\title{
PRODUCTION OF CARBON PRODUCTS USING A COAL EXTRACTION PROCESS DE-FC26-02NT41596
}

\author{
Semiannual Report \\ For the period: March 11, 2003--September 11, 2003 \\ Resubmitted: December 12, 2005 \\ Principal Investigator: \\ Dady Dadyburjor, $\mathrm{PhD}$ \\ Co-Investigators: \\ Chong Chen, $\mathrm{PhD}$ \\ Elliot B. Kennel \\ Liviu Magean \\ Peter G. Stansberry, PhD \\ Alfred H. Stiller, $\mathrm{PhD}$ \\ John W. Zondlo, PhD \\ West Virginia University \\ Department of Chemical Engineering \\ College of Engineering and Mineral Resources \\ PO Box 6102 \\ Morgantown WV 26505
}




\section{DISCLAIMER}

This report was prepared as an account of work sponsored by an agency of the United States Government. Neither the United States Government nor any agency thereof, nor any of their employees, makes any warranty, express or implied, or assumes any legal liability or responsibility for the accuracy, completeness, or usefulness of any information, apparatus, product, or process disclosed, or represents that its use would not infringe privately owned rights. Reference herein to any specific commercial product, process, or service by trade name, trademark, manufacturer, or otherwise does not necessarily constitute or imply its endorsement, recommendation, or favoring by the United States Government or any agency thereof. The views and opinions of authors expressed herein do not necessarily state or reflect those of the United States Government or any agency thereof. 


\section{Abstract}

High melting temperature synthetic pitches (Synpitches) were created using coal derivatives produced from a solvent extraction technique. Solvent extraction is used to separate hydrocarbons from mineral matter as well as other insolubles. Mild hydrogenation can be used to chemically modify resultant material to produce a true pitch. There are three main techniques which can be used to tailor the softening point of the Synpitch. First, the softening point can be controlled by varying the conditions of hydrogenation, chiefly the temperature, pressure and residence time in a hydrogen overpressure. Second, by selectively distilling light hydrocarbons, the softening point of the remaining pitch can be raised. Third, the Synpitch can be blended with another mutually soluble pitch or hydrocarbon liquid. Through such techniques, spinnable isotropic Synpitches have been created from coal feedstocks. Characteristics of Synpitches include high cross-linking reactivity and high molecular weight, resulting in carbon fibers with excellent mechanical properties. To date, mechanical properties have been achieved which are comparable to the state of the art achievable with conventional coal tar pitch or petroleum pitch. 


\section{Table of Contents}

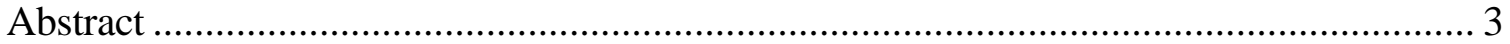

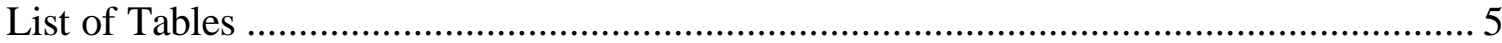

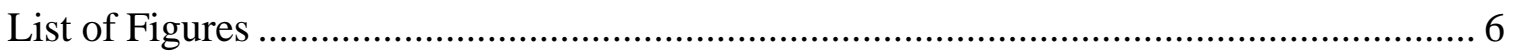

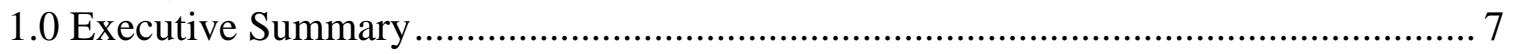

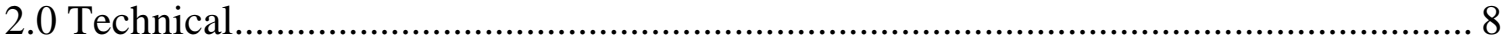

2.1 Isotropic and Mesophase-Based Fibers and Composites ….............................. 8

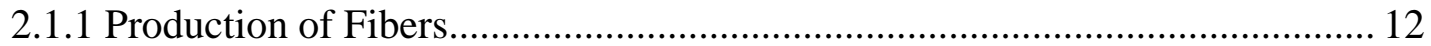

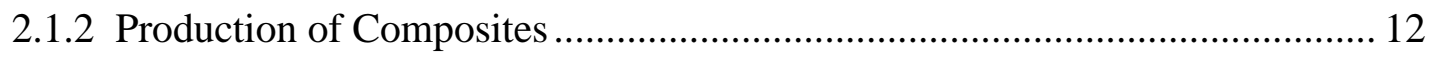

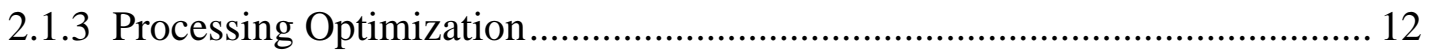

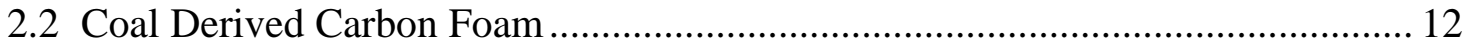

2.2.1 Pretreatment on Manufacture of Carbon Foams at Low Pressures................. 12

2.2.2 Evaluation of Properties of Foams............................................................ 12

2.3 Production of Coal-Derived Pitches and Cokes................................................. 13

2.3.1 Construction of a Continuous Green Coker ................................................... 20

2.3.2 Effects of Green Coking and Calcining Conditions .................................... 20

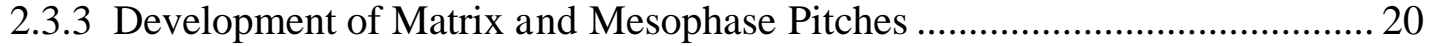

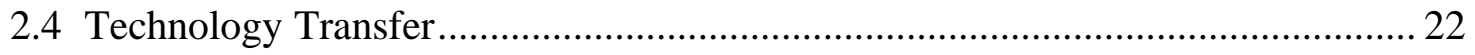

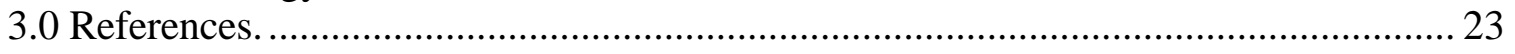




\section{List of Tables}

Table 1. Tensile Measurements of Synthetic Pitch Fiber ................................................ 9

Table 2. Tensile Measurements of Synthetic Pitch Fiber ............................................... 10

Table 3. Overall Mass Balances of the Coal Hydrogenation Reactions ........................... 21

Table 4. Reaction Conditions with Fresh Solvents ...................................................... 21

Table 5. Coal Conversion Efficiency......................................................................... 22 


\section{List of Figures}

Figure 1. SEM photomicrograph of Synpitch-Derived Carbon Fibers........................... 10

Figure 2. SEM photomicrograph of a highly irregular Synpitch-derived carbon fiber. .. 11

Figure 3. Stress-Strain Plot for a fiber derived from A-240/Bakerstown Synpitch. ........ 11

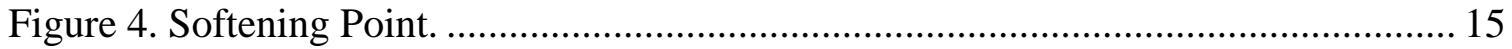

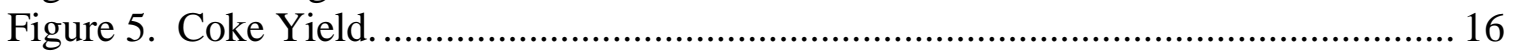

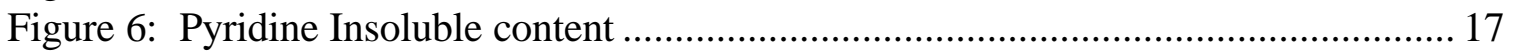

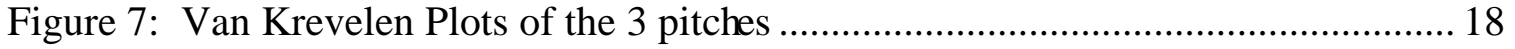




\subsection{Executive Summary}

The purpose of this DOE-funded effort is to develop technologies for carbon products from coal-derived feedstocks. Carbon products can include precursor materials such as solvent extracted carbon ore (SECO), synthetic pitch (Synpitch). In addition, derived products include carbon composites, fibers, foams and others. 


\subsection{Technical}

\subsection{Isotropic and Mesophase-Based Fibers and Composites}

This material was presented at the Pittsburgh Coal Conference, Synthetic Coal Pitch Derivatives for Spinning Carbon Fiber, Stephen P. Carpenter, Adam J. Berkovich (University of Kentucky Center for Applied Energy Research), James R. Bowers, Elliot B. Kennel, Liviu Magean, Peter G. Stansberry, and John W. Zondlo. This summarizes past work as well as current status.

High melting temperature synthetic pitches (Synpitches) were created using coal derivatives produced from a solvent extraction technique. Solvent extraction is used to separate hydrocarbons from mineral matter as well as other insolubles. Mild hydrogenation can be used to chemically modify resultant material to produce a true pitch. There are three main techniques which can be used to tailor the softening point of the Synpitch. First, the softening point can be controlled by varying the conditions of hydrogenation, chiefly the temperature, pressure and residence time in a hydrogen overpressure. Second, by selectively distilling light hydrocarbons, the softening point of the remaining pitch can be raised. Third, the Synpitch can be blended with another mutually soluble pitch or hydrocarbon liquid. Through such techniques, spinnable isotropic Synpitches have been created from coal feedstocks. Characteristics of Synpitches include high cross-linking reactivity and high molecular weight, resulting in carbon fibers with excellent mechanical properties. To date, mechanical properties have been achieved which are comparable to the state of the art achievable with conventional coal tar pitch or petroleum pitch.

Although most carbon fiber is currently spun using polyacrylonitrile (PAN) precursors, pitch based carbon fibers continue to find application because of their high modulus, low coefficient of thermal expansion, low electrical resistivity and high thermal conductivity. These fiber properties are influenced by the properties of the precursor pitch.

Synthetic coal derived pitches, or Synpitches, can be produced starting with raw coal by a combination of hydrogenation, separation and careful thermal processing in order to produce a tailored melting point pitch. In contrast to pitches obtained from coking ovens, synthetic coal pitch offers high cross-linking reactivity, high aromatic content, high molecular weight and tailorable properties. A potential cost advantage compared to PAN-derived fibers can be accrued due to the low cost of the feedstock material. Historically, however, processing costs have been consistently higher for pitchderived fibers. Variability of the feedstock is a major reason for higher processing and quality control costs of pitch fibers. Thus, a more uniform source of feedstock might result in reduced costs. With these objectives in mind, preliminary efforts have been carried out to produce carbon fibers using Synpitch.

Stansberry et al. produced isotropic synthetic pitch which was successfully spun into isotropic carbon fibers. ${ }^{1}$ Bituminous coal (WVGS 13421) was ground to -60 mesh and vacuum dried at $100{ }^{\circ} \mathrm{C}$ overnight. Six hundred grams of ground coal was mixed with 1.8 liters of tetralin, sealed and purged with hydrogen gas and pressurized to 400 psig. Mixing was accomplished using a magnetic stirrer at $1000 \mathrm{rpm}$. The reactor was 
heated for one hour at a maximum temperature of about $450{ }^{\circ} \mathrm{C}$ for one hour. The reactor was then cooled to ambient temperature, vented and opened. After washing with solvent, the product was centrifuged at $2000 \mathrm{~g}$ for one hour to separate solid material (principally mineral matter and undissolved coal) from the liquefied coal. Typical yields ranged from 70 to $80 \%$ by mass.

The softening point of the resultant pitch was raised by a combination of thermal processing and vacuum distillation. Fibers were spun by the University of Kentucky Center for Applied Energy Research, using a single hole spinneret. Stabilization occurs via a temperature ramp of $0.05{ }^{\circ} \mathrm{C} / \mathrm{min}$ to $310{ }^{\circ} \mathrm{C}$. Finally, carbonization was accomplished in a separate furnace with a $20{ }^{\circ} \mathrm{C} / \mathrm{min}$ ramp to $1100{ }^{\circ} \mathrm{C}$. The result is an isotropic carbon fiber.

Initial results, based on measurements made by an independent contractor, seemed to indicate that strengths as high as $1.3 \mathrm{GPa}$ had been attained. However, these data were not duplicated using ASTM D3379. Measurements made by both the University of Kentucky Center for Applied Energy Research (CAER) and West Virginia University (WVU) indicated a significantly lower value, as shown in Table 1. Both data sets indicate a lower strength value than the earlier estimate. In addition, the measured modulus is about an order of magnitude lower than commercially available pitch fibers.

Table 1. Tensile Measurements of Synthetic Pitch Fiber

\begin{tabular}{|l|l|l|}
\hline Property & CAER measurement & WVU measurement \\
\hline Diameter & $31.9+/-1.8$ microns & $32.7+/-2.0$ microns \\
\hline Strength & $519+/-59 \mathrm{MPa}$ & $437+/-51 \mathrm{MPa}$ \\
\hline Modulus & $38.7+/-1.6 \mathrm{GPa}$ & $37.9+/-5.3 \mathrm{GPa}$ \\
\hline Strain & $1.34+/-0.13 \%$ & $1.16+/-0.16 \%$ \\
\hline
\end{tabular}

An alternate method also investigated for enhancing the properties of low softening point pitches. In this case the motivation is cost reduction by utilizing lower cost precursors, as well as improving the ease of stabilization processing. For example, Ashland petroleum pitch A-240 can be spun into fiber, but is usually impossible to stabilize owing to its low softening point $\left(240{ }^{\circ} \mathrm{F}\right.$ or $\left.116{ }^{\circ} \mathrm{C}\right)$. By combining this lowsoftening-point pitch with Solvent Extracted Carbon Ore (SECO), it was hoped that the resultant mixture would have a softening point appropriate for fiber spinning, as well as adequate properties after stabilization.

SECO is produced by dissolving ground coal in a solvent such as n-methyl pyrrolidone (NMP). For these experiments, coal from the Lower Bakerstown seam was used. After centrifugation to remove solids (chiefly mineral matter and undissolved carbon), the resultant coal solution is reconstituted and dried.

SECO was combined with A240 pitch to create a solution was $10 \%$ SECO by mass. The resultant pitch was spun into fiber and stabilized.

Although stabilization was considered successful, the resultant fibers were brittle and contained significant defects, as shown in the SEM photomicrographs in Figures 1 and 2. The photomicrographic analysis reveals extensive formation of pores within the body of the fibers, probably due to devolatilization during stabilization and calcining. Raw Instron data is shown in Figure 3. Mechanical property measurements are listed in Table 2. 
Future experiments are planned to modify the protocol in order to reduce this problem, in which case superior mechanical properties are expected.

Table 2. Tensile Measurements of Synthetic Pitch Fiber

\begin{tabular}{|l|l|}
\hline Property & Measured Value \\
\hline Diameter & $35.9+/-6.9$ microns \\
\hline Strength & $237+/-90 \mathrm{MPa}$ \\
\hline Modulus & $33.6+/-9.3 \mathrm{GPa}$ \\
\hline
\end{tabular}

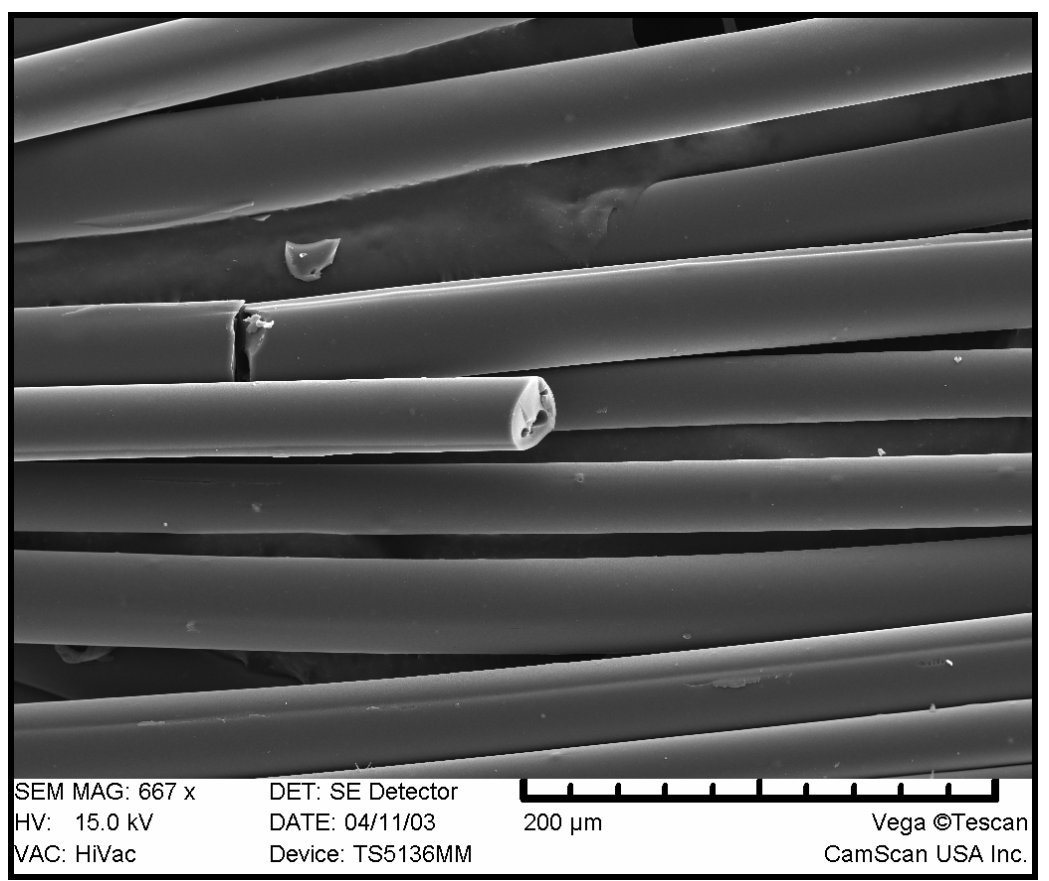

Figure 1. SEM photomicrograph of Synpitch-Derived Carbon Fibers, showing defects and irregular surfaces. 


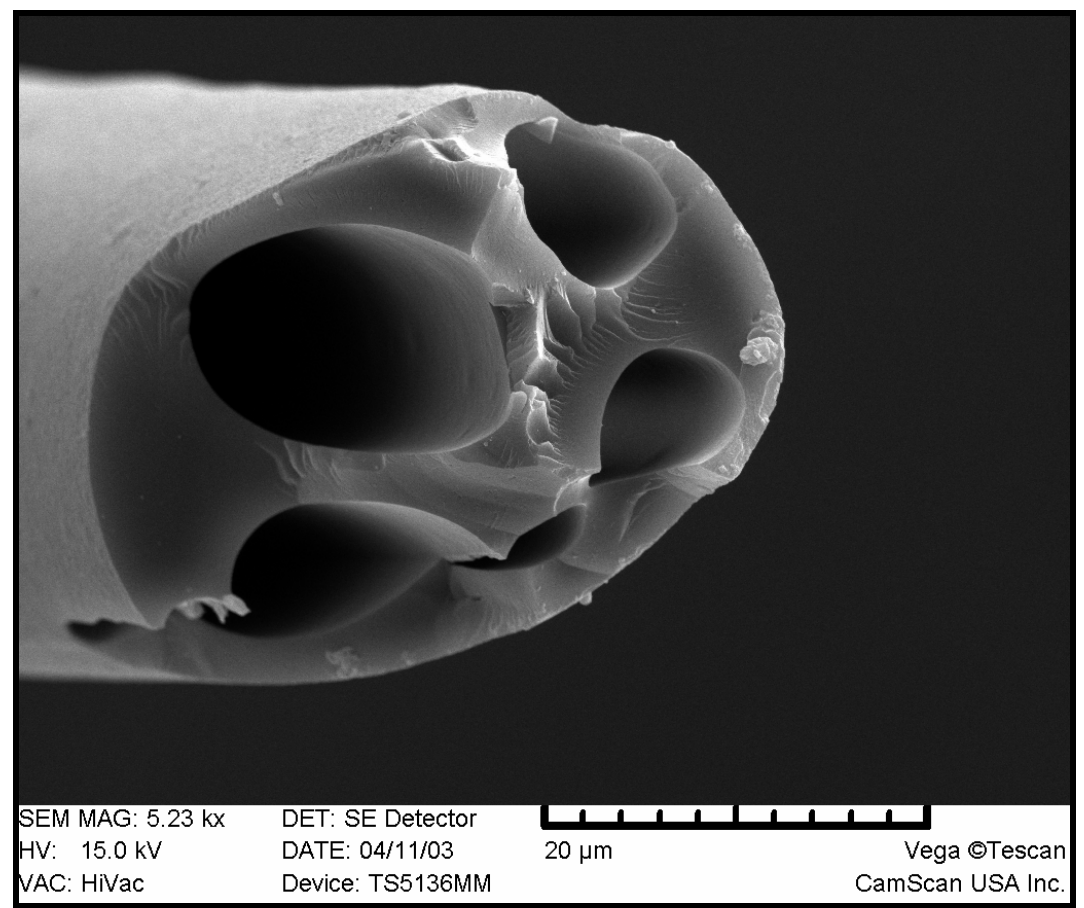

Figure 2. SEM photomicrograph of a highly irregular Synpitch-derived carbon fiber (courtesy Vega).

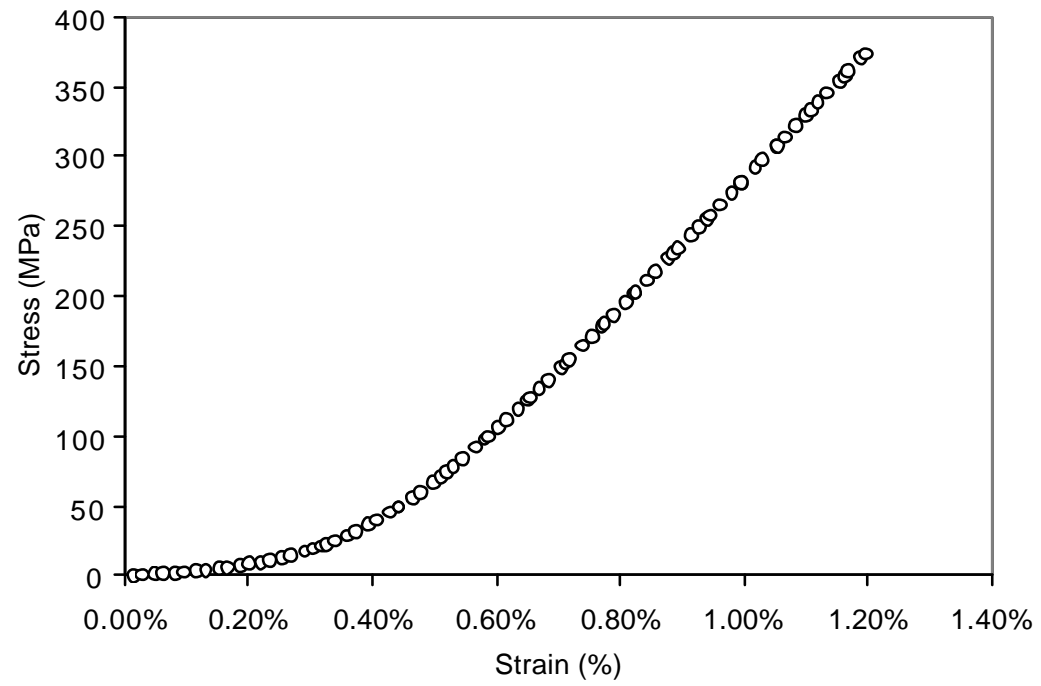

Figure 3. Stress-Strain Plot for a fiber derived from A-240/Bakerstown Synpitch.

In summary, the production of carbon fibers from synthetic coal pitch (Synpitch) as well as from a blend of petroleum pitch augmented with solvent extracted carbon ore (SECO) was demonstrated. Fiber from pure Synpitch demonstrated strength comparable to commercial grade fibers. Future trials will be made using anisotropic pitches now being produced on benchtop scale, and are expected to demonstrate improved strength and especially modulus. 
On the other hand, fibers produced from blended pitch exhibited significant nonuniformity, including the presence of pores within the calcined fiber. These defects are potentially correctable, but acceptable fibers have not yet been produced using blended pitch feedstock. Future efforts will modify the feedstock as well as the stabilization protocol to attempt to remedy this difficulty.

\subsubsection{Production of Fibers}

No data this reporting period.

\subsubsection{Production of Composites}

No data this reporting period.

\subsubsection{Processing Optimization}

No data this reporting period.

\subsection{Coal Derived Carbon Foam}

\subsubsection{Pretreatment on Manufacture of Carbon Foams at Low Pressures}

Several coal samples were prepared for foam making, including 30 pounds of Sewell and Kingwood coal which was ground and sieved into different size fractions (50100mesh, 100-200 mesh and <200mesh).

Over 20 pieces of carbon foam were produced from Kingwood coal using high pressure foaming method, and final machined to create general purpose specimens..

Condensation reactions of pitch materials were investigated in order to be able to better tailor the properties of precursors for carbon foam production. Pitch samples included Petroleum pitch and coal based synpitch. Condensation was carried out under $\mathrm{N} 2$ at a temperature range of 350 to $400^{\circ} \mathrm{C}$ for different treatment times.

\subsubsection{Evaluation of Properties of Foams}




\subsection{Production of Coal-Derived Pitches and Cokes}

The following work was presented at the $5^{\text {th }}$ AFRL Carbon Foam Workshop: "Synthetic Pitch Feedstocks for Carbon Foam," Mitchell Clendenin, Elliot B. Kennel, Nathan King, and Peter G. Stansberry.

The properties of carbon foams are highly dependent upon the properties of the pitch precursor used to fabricate the foam. The properties of pitches, whether coaltarderived, petroleum-derived, or synthetic, can be significantly modified with thermochemical treatments. As a result, properties such as softening point, viscosity coke yield, solids content and others can be modified.

The main industrially produced pitches come from the petroleum refining industry (petroleum pitch) and metallurgical coking industry (coal tar pitch).

During crude oil is distilled into three major fractions, referred to as the light, middle, and heavy fraction. The heavy fraction is then distilled further to yield heavy oils and a solid residue. The leftover solid residue in the bottom of the still is petroleum pitch.

Coal tar pitch is produced as a byproduct of metallurgical coking using coal. The coal is heated in the absence of air, resulting in devolitalization of low molecular weight hydrocarbons and other trapped gases and vapors. Condensing volatiles results in the formation of coal tar. The coal tar is then distilled to remove all of the heavy oils, and the remaining product is called coal tar pitch.

A third type of pitch is referred to as Synthetic Pitch or Synpitch, which is created using chemical synthesis techniques. This technology is currently being employed at Mitsubishi Gas Company to produce their Mitsubishi pitch starting For example, Mitsubishi AR Pitch is produced from naphthalene being reacted over a catalyst. Starting from a specific feed composition ensures that the product will always be the same. At West Virginia University, another type of synthetic pitch is currently being produced, which is referred to as SynPitch. This pitch is the result of coal hydrogenation and solvent extraction. SynPitch slightly varies from batch to batch since coal composition is dependent on the location of the mine, and can even vary within the same mine. This is also the case for petroleum pitch and coal tar pitch, since petroleum and coal composition differ depending on where they are found.

Regardless of origin, the two main families of pitch used in industry today are isotropic pitch and anisotropic pitch. Isotropic pitch has the same properties throughout the pitch and does not vary in any direction. There is no real molecular order in an isotropic pitch. Uses for isotropic pitch include general purpose carbon fibers (GPCF), sacrificial carbon anodes used for aluminum production, and others. Carbon foam produced from isotropic feedstocks does not achieve graphitic order, and hence can not achieve high thermal and electrical conductivity, even upon high temperature heat treatment.

Conversely, highly anisotropic pitch, such as a mesophase pitch, can result in the formation of graphite upon heat treatment. In pitch form, molecular order occurs as liquid crystals of carbonaceous mesophase. Slow thermal treatment and higher temperatures can result in an increased number of these crystals that start to join together into a bulk mesophase. In the fiber industry mesophase pitches are mainly used for high 
performance carbon fibers (HPCF) with high modulus, high thermal conductivity and low electrical resistivity.

Production of low-cost, high-quality mesophase pitch remains a key goal for the materials community, with significant implications for producers of carbon and graphite foams.

\section{Mesophase Pitch Production}

Isotropic pitch or raw coal can be converted to anisotropic pitch through thermochemical processing to create additional aromatic hydrocarbons. This can be accomplished by a mild hydrogenation process, usually with an intermediate donor solvent, such as tetralin produced by hydrogenation methyl naphthalene.

This process is accomplished in a pressurized high temperature reactor. Typically a pressure of roughly $500 \mathrm{psi}$ and a temperature of $400 \mathrm{C}$ are required to break and reform the chemical bonds in the pitch to form aromatics. Hydrogenation of coal to a level of 3-5 weight percent additional temperature is sufficient to produce aromaticity. Centrifugation or filtration is required to remove insoluble material, thus resulting in true pitch. Thus, this is considered to be a solvent extraction technique, although typically the hydrogen donor also serves as the solvent.

Additional thermal and light chemical processing is used to remove light hydrocarbons, further modifying the pitch and inducing the formation of mesophase.

Chemical treatment can be done in a variety of ways. These chemical treatments cause irreversible changes to the pitch composition. Chemical methods of increasing the softening point and coke yield include the use of hydrogenation agents such as elemental sulfur, carbon black, and additives such as $\mathrm{FeCl}_{3}$ and $\mathrm{AlCl}_{3}$, as well as the use of catalytic polymerization techniques.

Softening point is a key parameter in determining the optimum temperature for foaming. Pitches exhibit a broad softening range rather than melting at a specific temperature. The softening point is determined by the molecular weight of the components in the pitch. Pitches with a low average molecular weight generally have a lower softening point than a pitch containing heavy molecular compounds. 
Three diverse pitches were used to demonstrate the ability to modify the softening point. A-240 is a petroleum pitch from Ashland Oil. A commercial coal tar pitch was obtained from Koppers Industries. Finally a synthetic pitch was produced using hydrogen coal at WVU.

The first table demonstrates how the softening point of any of the three pitches can be altered. A higher softening point will make the pitch a better candidate for fiber spinning. Longer treatment times will result in a higher softening point.

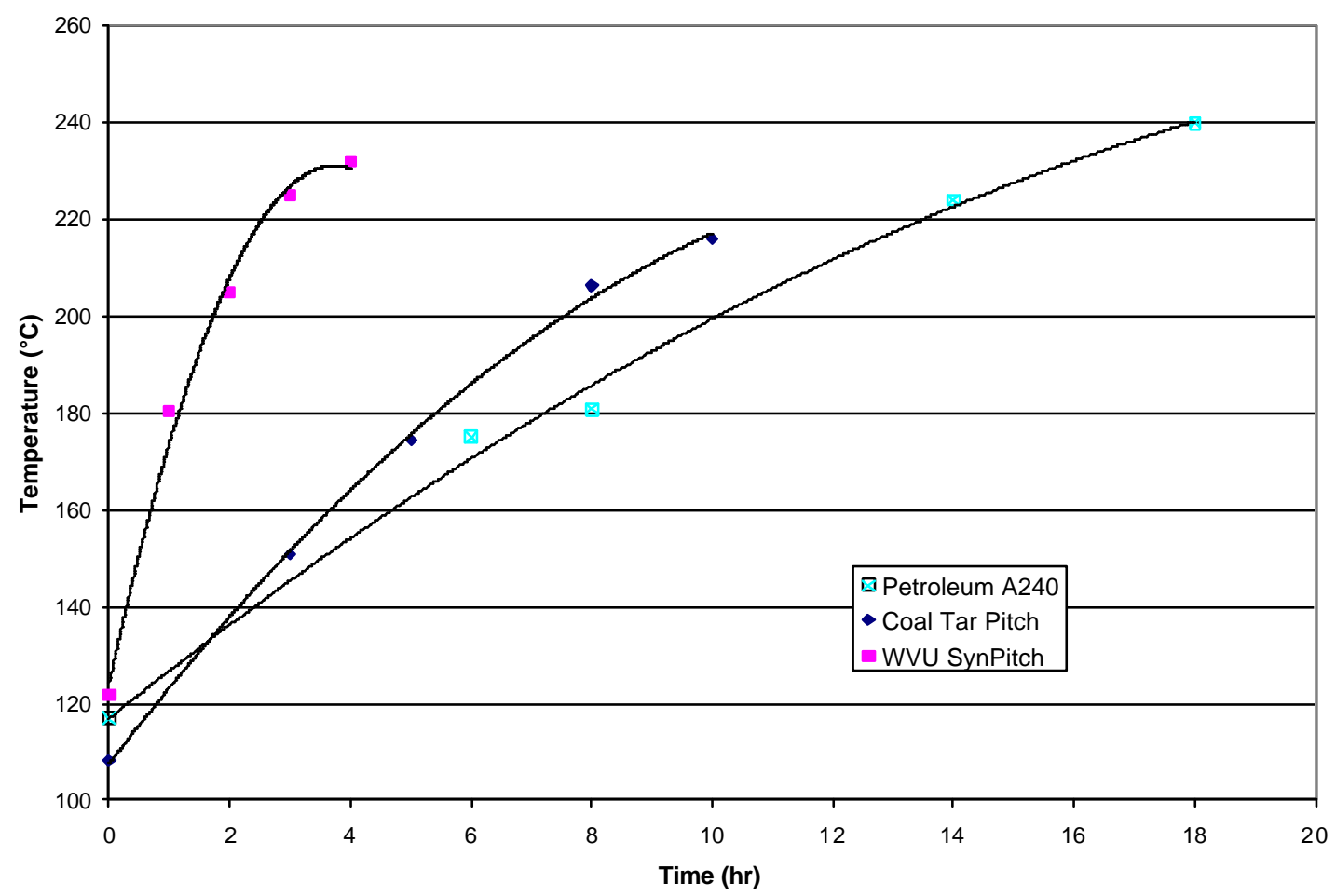

Figure 4. Softening Point. 
Additionally, the coke yield of a particular pitch can be altered in a similar fashion. A pitch with a higher coke yield has a higher concentration of carbon compared to the other constituents.

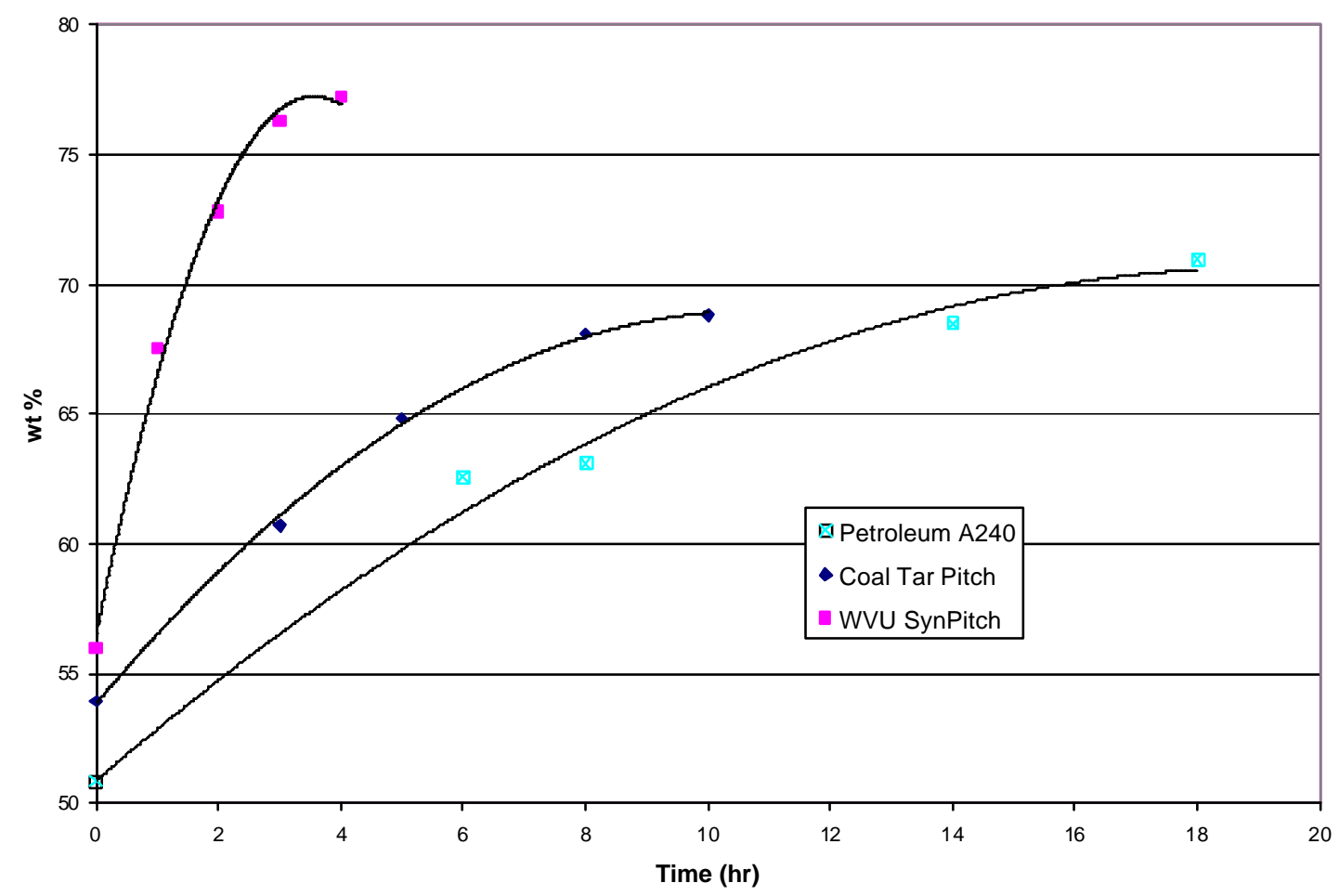

Figure 5. Coke Yield.

The solids content of pitches can play a very vital role in determining for what end use the pitch is suitable. One measure of this is the Pyridine Insolubles. The pitch undergoes a typical extraction, only using Pyridine rather than the usual solvents (Toluene, THF, etc.). As the PI increases, the solids content increases. This is due to more and more of the compounds in the pitch becoming larger and more poly-aromatic. Again, the treatment method used in this series of experiments shows an ability to increase the \%PI of a particular pitch. 


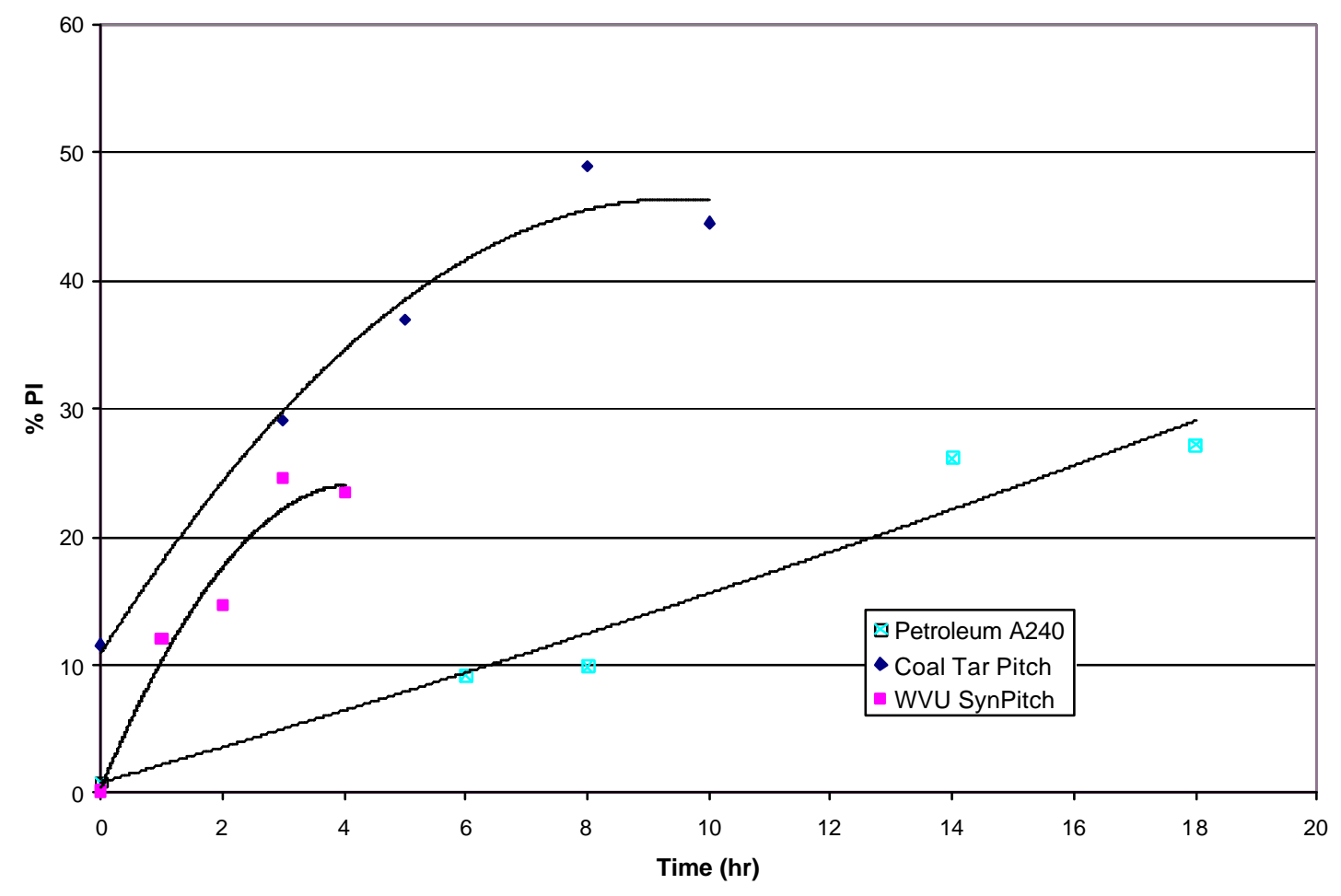

Figure 6: Pyridine Insoluble content

As can be seen from the Van Krevelen plots in Figure 7, the three pitches exhibit three different types of reaction mechanisms when processed. The petroleum pitch, A240, goes through a dealkylation process while the coal tar pitch goes through a dehydroxylation mechanism. The WVU Synpitch goes through both processes of dealkylation and dehydroxylation. In looking at these results, it can be seen that every type of pitch acts differently. Using our processing technique, these pitches can easily be tailored to meet the specifications for the ideal applications using the pitch. 


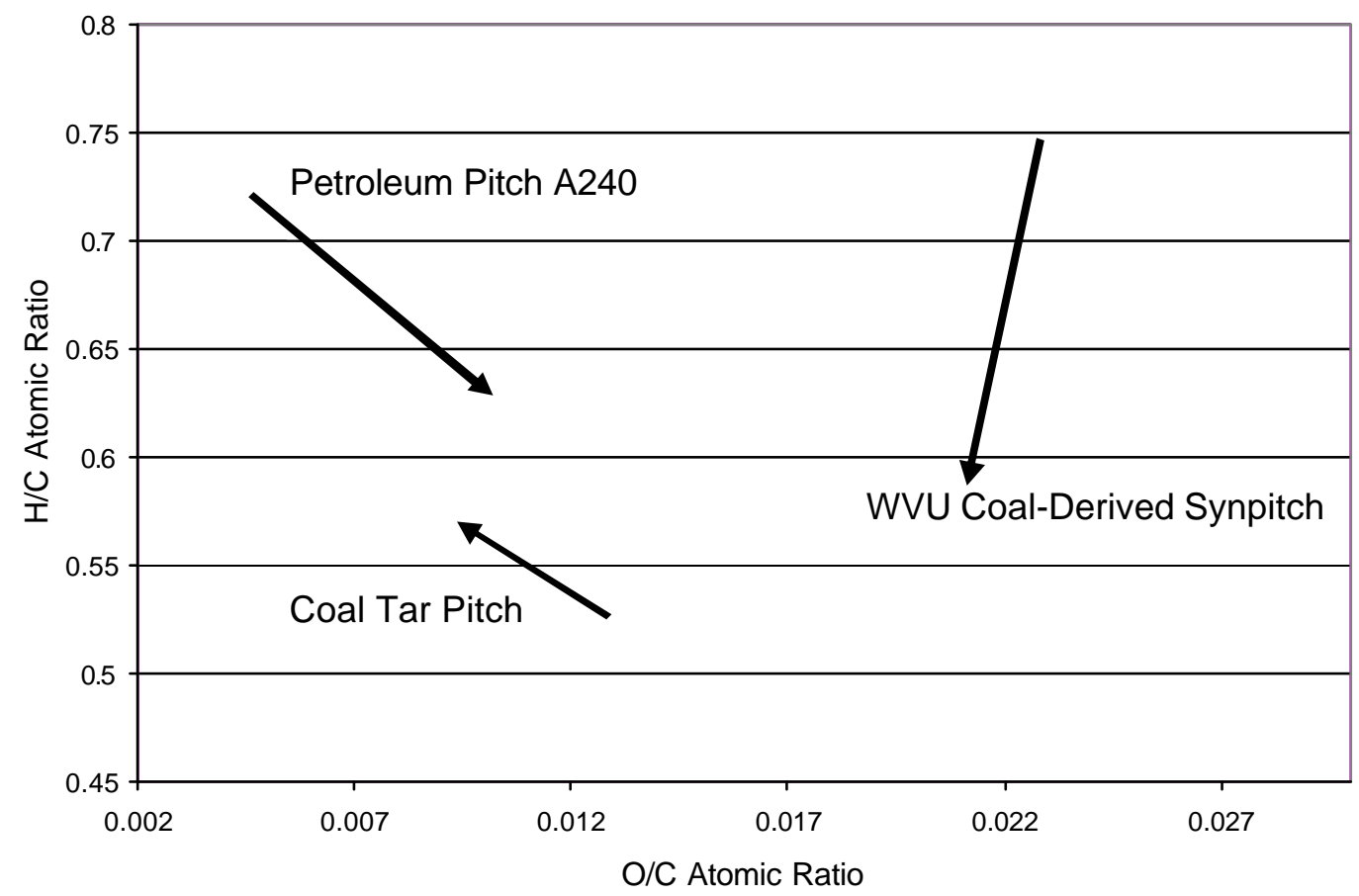

Figure 7: Van Krevelen Plots of the 3 pitches

Properties of pitches of all kinds can be effectively controlled using the proper processing techniques. This opens the door to a large field of uses, including the carbon foam industry. Carbon foams can be made from these pitches, and depending on what type of foam is needed, the pitch can be treated to give the ideal starting material.

\section{Hyrdrogenation Notes}

After an unsuccessful pressure test of 1-gallon autoclave reactor in ERB Rm 317, it was decided that, pending repair of an inadequate seal, the solvent hydrogenations would be carried out in a 5 gallon reactor. To avoid overproducing hydrogenated solvent, the reactor would be filled only approximately half full.

A new annular catalyst basket was constructed using SS 316 with an inner diameter of 6.0 inches and an outer diameter of 7.5 inches and a height of 8 inches. The basket was adjusted to ensure both stability and impeller clearance.

\section{Catalyst preparation}

Once the basket was completed, $2.5 \mathrm{lb}$ of a $\mathrm{NiMo}$ catalyst was poured into the annular basket. The 5-gallon reactor was pressurized to $1000 \mathrm{psig}$ with $\mathrm{N}_{2}$ and found to be leaktight. Approximately $250 \mathrm{~mL}$ of DMSO (dimethyl sulfoxide) was placed into the reactor. This amount was calculated from the literature made available to us from the manufacturers, Criterion Catalyst. 
The catalyst was sulfided for 2 hours at a temperature of $210^{\circ} \mathrm{C}$ at atmospheric pressure with the impeller spinning at $1000 \mathrm{rpm}$. The hot gases were vented from the 5gallon reactor and the reactor was flushed out with $\mathrm{N}_{2}$ gas.

\section{Elution Strengths of Solvents}

An Excel Spreadsheet was developed to estimate elution strengths of different solvents on alumina. This spreadsheet allowed calculation of solvent ratios necessary to provide small and smooth increases in solvent elution strengths from non-polar to polar solutions. Nineteen different mixtures of solutions will be created from the following materials:
a. Hexane
b. Toluene
c. Tetrahydrofuran
d. Dimethylsulfoxide
e. 2-Methoxyethanol
f. Ethanol

Carbontetrachloride was eliminated from the solvent list by using equivalent mixtures of hexane and toluene.

The idea of using a LVDT (Linear Variable Differential Transducer) to measure coal swelling was explored. The ultrasonic mixer is warmed up and ready.

Almost all UV-VIS spectroscopy data is now collected. Some problems with the UV-VIS spec machine have been encountered and Bob Tarhi at Perkin Elmer is helping us to work them out.

\section{Carbon Black Base Oil}

A 55-gal drum of CBB (Carbon Black Base or Creosote Oil) from Koppers in the high-bay was fitted with the drum warming straps and a motorized mixer. A handcranked pump was obtained to extract two 5-gallon samples from the drum. After a few minor mishaps, the drum's contents were heated and we anticipate extracting samples for hydrogenation.

\section{Hydrotreating Facility}

The plumbing in the bunker is complete save for the nitrogen line for the actuated valve. The heater bands are in place as well. Nine of the twelve heater bands on the lines. 
Several runs have been made with SECO. Ash content, originally measured at $2 \%$ are now being measured at much less than $1 \%$. This is attributed to residual rust in the system, which is slowly being swept out of the system (which has been rebuilt with mainly stainless steel and chrome plated components to avoid contamination issues, which we believe are corrosion-related).

However, on some occasions, the piping to the centrifuge becomes plugged as the deashing process is initiated. A possible solution to this is to flush the lines with NMP between runs to remove particulates and silt that are believed to be accumulating in the piping between the reactor and the centrifuge. Also in the high bay a system to heat and stir materials in a 55 gallon steel drum is being trialed. A nearly homogeneous solution in the drum was successfully produced. Similar material will be used for hydrogenation in the near future.

\subsubsection{Construction of a Continuous Green Coker}

The Coking Unit is under construction at Northco Industries. No other data in this reporting period.

\subsubsection{Effects of Green Coking and Calcining Conditions}

No data in this reporting period.

\subsubsection{Development of Matrix and Mesophase Pitches}

A mass balance was performed for each hydrogenation reaction trial. The components of the mass balance were the amount of coal and solvent charged into the reactor and the amount of pitch, THF insolubles (residue), and recycle solvent collected after processing the hydrogenation products. The results of the mass balances can be seen in Table 2. The conditions for each hydrogenation run are given in Table 4.

For every reaction, a negative mass balance was observed, indicating a net loss of mass during the reaction and product separation steps. There are several different explanations for the negative mass balances. First, any product gas that may have formed during the reactions was not collected and quantified and thus is absent from the mass balance calculation. Second, any light material that was produced during the hydrogenation reaction that condensed upon quenching of the reaction could have been lost during the vacuum distillation step. Any light material which did not collect with the other recycle solvent but would rather be collected in the cold trap of the vacuum pump. This was confirmed by periodically checking the cold trap of the vacuum pump for the presence of collected liquid (small amounts of liquid were usually present). Finally, the process of separating the hydrogenation products into different fractions (pitch, residue, and recycle solvent) required a significant amount of experimental transfers and procedures. Each of these procedures resulted in some amount of mass loss. 
For the majority of the reaction runs listed in Table 3 the amount of mass loss was around $7 \%$ by weight or less for fresh solvents. This is a reasonable result considering the procedures that were used in obtaining the product fractions.

Table 3. Overall Mass Balances of the Coal Hydrogenation Reactions with Fresh Solvents

\begin{tabular}{||l|l|l|l|l|l|l|l|l|l|l||}
\hline Run $^{\mathrm{a}}$ & Trial & $\begin{array}{l}\text { In } \\
\text { Coal }(\mathbf{g})\end{array}$ & $\begin{array}{l}\text { In } \\
\text { Solvent } \\
\mathbf{( g )}\end{array}$ & $\begin{array}{l}\text { Total } \\
\text { In (g) }\end{array}$ & $\begin{array}{l}\text { Out } \\
\text { Pitch }(\mathbf{g})\end{array}$ & $\begin{array}{l}\text { Out } \\
\text { THF Ins. } \\
(\mathbf{g})\end{array}$ & $\begin{array}{l}\text { Out } \\
\text { Rec. } \\
\text { Solv. (g) }\end{array}$ & $\begin{array}{l}\text { Total } \\
\text { Out (g) }\end{array}$ & $\begin{array}{l}\text { In - Out } \\
(\mathbf{g})\end{array}$ & $\begin{array}{l}\text { Loss } \\
(\%)\end{array}$ \\
\hline 1 & A & 4.001 & 20.02 & 24.021 & 2.49 & 2.3119 & 17.65 & 22.452 & 1.569 & 6.5 \\
\hline 1 & B & 4.0032 & 20.1 & 24.1032 & 3.01 & 2.4008 & 16.87 & 22.2808 & 1.8224 & 7.6 \\
\hline 2 & A & 4.002 & 20.2 & 24.202 & 2.71 & 2.4201 & 17.22 & 22.3501 & 1.8519 & 7.6 \\
\hline 2 & B & 4.0056 & 20.3 & 24.3056 & 2.967 & 2.371 & 17.29 & 22.628 & 1.6776 & 6.9 \\
\hline 3 & A & 4.0001 & 20.3 & 24.3001 & 3.94 & 2.8429 & 15.87 & 22.6529 & 1.6472 & 6.7 \\
\hline 3 & B & 4.0032 & 20.1 & 24.1032 & 3.98 & 2.8156 & 15.1 & 21.8956 & 2.2076 & 9.1 \\
\hline
\end{tabular}

${ }^{\mathrm{a}}$ Here run 1 is using $\mathrm{CBB}$, run 2 is using $\mathrm{HCO}$ and run 3 is using $\mathrm{RCO}$

Table 4. Reaction Conditions with Fresh Solvents

\begin{tabular}{|c|c|c|c|c|c|}
\hline Run & Solvent & $\begin{array}{c}\text { Solvent/Coal } \\
\text { Ratio }\end{array}$ & $\begin{array}{c}\text { Temperature } \\
{ }^{\mathbf{0}} \mathbf{C}\end{array}$ & Pressure Psig & $\begin{array}{c}\text { Time } \\
\text { Hr }\end{array}$ \\
\hline 1 & CBB & 5 & 400 & 500 & 1 \\
\hline 1 & CBB & 5 & 400 & 500 & 1 \\
\hline 2 & HCO & 5 & 400 & 500 & 1 \\
\hline 2 & HCO & 5 & 400 & 500 & 1 \\
\hline 3 & RCO & 5 & 400 & 500 & 1 \\
\hline 3 & RCO & 5 & 400 & 500 & 1 \\
\hline
\end{tabular}

Similarly a mass balance will be performed on recycle solvents and the n can be compared with the percent loss obtained with fresh solvents.

\section{Additional Hydrogenation Trials}

Carbon black base (CBB) oil, a coalderived oil which we intend to consider as a low-cost hydrogenation solvent, was hydrogenated using $\mathrm{Ni}$ - Mo catalyst using a catalyst basket. Ni - Mo catalyst is taken in the oxide form is first sulfided using di-methyl sulfoxide ((DMS) and then hydrogenated at $350^{\circ} \mathrm{C}$.

Hydrogenation reactions were accomplished using the raw $\mathrm{CB}$ and the hydrogenated $\mathrm{CBB}$ under the following conditions, with results summarized in Table 5:

a. Solvent: CBB

b. $\mathrm{H}_{2}: 500 \mathrm{psi}$ 
c. Temperature: $400^{\circ} \mathrm{C}$

d. Solvent/ coal ratio: $5 / 1$

Table 5. Coal Conversion Efficiency

\begin{tabular}{|c|c|l|l|l|l|l|l|l|}
\hline S.No & Solvent & $\begin{array}{l}\mathrm{H}_{2} \\
\text { pressure, } \\
\text { psig }\end{array}$ & $\begin{array}{l}\mathrm{N}_{2} \\
\text { pressure, } \\
\text { psig }\end{array}$ & $\begin{array}{l}\text { Temp, } \\
{ }^{\circ} \mathrm{C}\end{array}$ & $\begin{array}{l}\text { Solvent/ } \\
\text { coal ratio }\end{array}$ & $\begin{array}{l}\text { Amount } \\
\text { of coal } \\
\text { used (g) }\end{array}$ & $\begin{array}{l}\text { Amount } \\
\text { of un- } \\
\text { converted } \\
\text { coal (g) }\end{array}$ & $\begin{array}{l}\text { Con- } \\
\text { version, } \\
\%\end{array}$ \\
\hline 1 & CBB & 500 & & 400 & 5 & 4.0041 & 1.8581 & 57.50 \\
\hline 2 & CBB & 500 & & 400 & 5 & 4.2406 & 1.9836 & 57.15 \\
\hline 1 & $\begin{array}{c}\text { CBB } \\
\text { hydrogen } \\
\text { ated) }\end{array}$ & 0 & 500 & 400 & 5 & 4.0015 & 1.8591 & 61.01 \\
\hline 2 & $\begin{array}{c}\text { CBB } \\
\text { hydrogen } \\
\text { ated) }\end{array}$ & 0 & 500 & 400 & 5 & 4.0023 & 1.7279 & 61.02 \\
\hline
\end{tabular}

The results show that hydrogenated $\mathrm{CBB}$ oil is at least equally as effective as fresh $\mathrm{CBB} / \mathrm{H}_{2}$ and that coal can be converted without the need for a high hydrogen overpressure. This suggests that modifications can be made to the baseline hydrotreating and solvent extraction procedure.

\subsection{Technology Transfer}

No data this reporting period. 


\subsection{References.}

${ }^{1}$ P. G. Stansberry et. al, Production of Fibers and Composites from Coal Based Precursors, DE-FC26-98FT40350. 